\section{What computational sciences can do for your heart}

Michèle Barbier and Maxime Sermesant Inria Sophia Antipolis - Méditerranée, France Oscar Camara

Universitat Pompeu Fabra (UPF), Spain Yves Coudière

Université de Bordeaux, Institut de Rythmologie et modélisation cardiaque, France

Beatriz Trenor

Universitat Politècnica de València - Centro de investigacion e innovacion en bioingenieria, Spain

Most people know someone suffering from cardiovascular disease. There are 15 million people in Europe currently living with heart failure. Not only does the condition lead to a lasting and significant impact on physical health, but it also affects overall well-being, manifesting in issues such

as anxiety or mood swings.
Unlike other cardiovascular diseases, the prevalence of heart failure continues to rise. While it is clear that solution are required, it is especially difficult to find new drugs or devices for this disease. Despite significant investmen in healthcare, the number of new dru approvals is not increasing. Why? Because of barriers such as the high cost of R\& regulatory complexity and difficulty commercialising new products.

\section{Digital transformation}

The power of digital technology and data exchange is known to support innovation: artificial intelligence (Al) high-performance computing, clou computing, and the internet of things Our heath deta is no excerton. It Our heath data is no exception. It can significantly contribute to better disease prevention, early diagnosis, and the evelopment of a personalised service.

n-silico methods are computationa predictions-in-silico referring to the silicon in our computers. These in-silico methods can make fast prediction for large sets of compounds in a hig throughput mode.

In-silico computational approaches can be used to predict complex clinical scenarios.

Health care and digita innovation in Europe

The European Commission (EC) supports using these new technologies and health data in the digital transformation of health and care. Hence the EC is funding SimCardioTest to demonstrate the feasibility, efficacy and benefits of in-silico trials for disease. Specifically, SimCardioTest aims to provide insight into designing new predictive tools in cardiac pathologies and accelerate the uptake of computer simulations for testing medicines and medical devices.
SimCardioTest

SimCardioTest has been funded for four years (2020-24) to create a secure, standardised cloud-based platform that allows seamless in-silico trial runs. Its goal is to win the trust of patients, scientists, regulators, physicians and other healthcare professionals and promote innovation in Europe and elsewhere.

The project brings together outstanding expertise in European computer modelling, simulation, and medical devices. There are ten project partners led by the Intitut Nationelde Recherche en Informatique et Autom arigue, France. The other partners are Universie de The other partners are Universite de Pompeu Fabra (UPF) and Universitat Polipen Fabra (UPF) and Universiet Poltecrica de Valencla, (UPV), Spain; Simula Research laboratory AS (SRL), Norway, InSilicoThals Technologies SPA (IST), Italy, SORIN CRM SAS (MPC) and ExactCure (EXC), France; Boston Scientific Scimed Inc (BSC), United States; and the Virtual Physiological Human Institute for Integrative Biomedical Research VZW (VPHi), Belgium.

The partners are supported by an advisory board composed of the European Agency for Medicine, the Food and Drug Administration in the United States, the University of Lyon, Dassault Systèmes, the pharmaceutical company

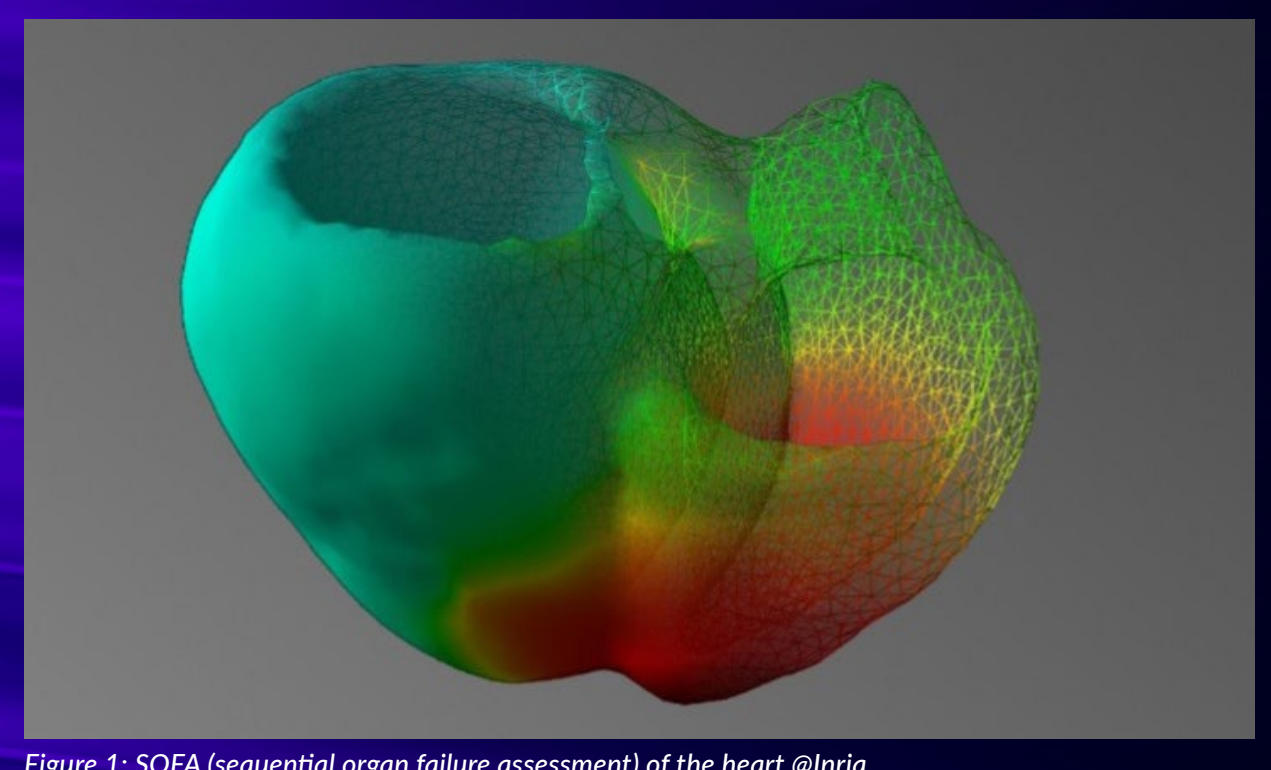

Figure 1: SOFA (sequential organ failure assessment) of the heart @Inria.
Roche, and Auckland Bioengineering stitute in New Zealand.

\section{SimCardioTest will}

demonstrate the benefit

of in-silico trials testing

three use cases in

cardiology.

\section{How is a simulation}

\section{obtained?}

ew predictive tools can now be created thanks to access to high-performance computing infrastructures (HPC), patient-specific information, and big data analysis based upon Al. Computational simulation is based on the deep analysis of medical images such as $3 \mathrm{D}$ computed tomography (CT) scans, echography and magnetic resonance images. These images are processed with $\mathrm{Al}$ tools to create a 3D model that is specific for each patient (Figure 1). some characteristics of a patient's eart, with their specific morphology,

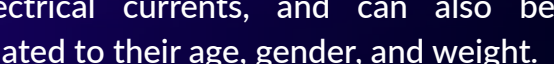
These computational simulations Tor personalised, adapted medicine. 


\section{Three use cases as \\ demonstrators in}

cardiac pathologies

To test drugs and devices, specific computational models will be created for cardiac pathologies such as ischaem atrial fibrillation, and heart failure.

\section{Pacing devices used in}

\section{cardiac arrhythmias associated} with heart failure

With electrical impulses regulated by specific heart cells, the heart beats synchronously. A pacemaker or defibrillator (a small battery-powered device) will help maintain a normal heart
rhythm and effective heart contractions rhythm and effective heart contraction
when your heart beats asynchronously when your heart beats asynchronously (arrhythmia, fibrillation). These devices by a flexible wire to small electrodes anchored in the heart muscle (Figure 2). The electrodes deliver electrical impulses to the heart that are generated and controlled the by pacemaker.

SimCardioTest's objective is to create computational models of the navigation of the lead from its insertion point towards its final location in the heart and evaluate its pacing properties at this location. In the models, the risks of perforation and rupture of the heart from
the fatigue of leads will be evaluated, as well as the threshold of energy required for the stimulation of a heartbeat for typical designs of the device. This work will impact the future design pacemakers or defibrillation devices.

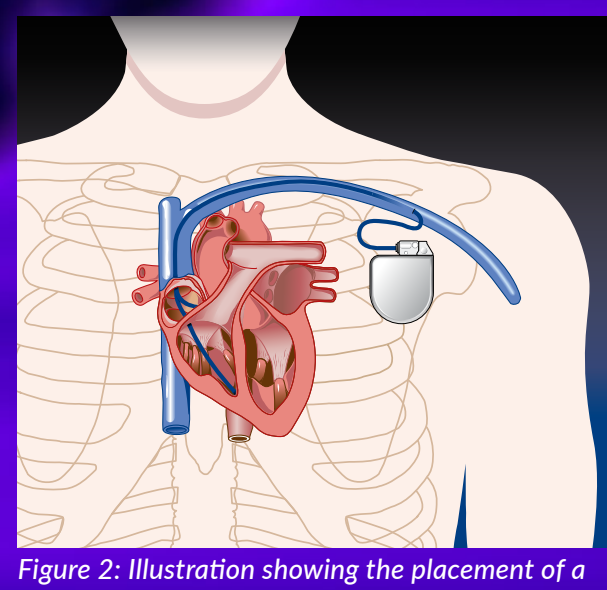
pacemaker that is fitted to a heats

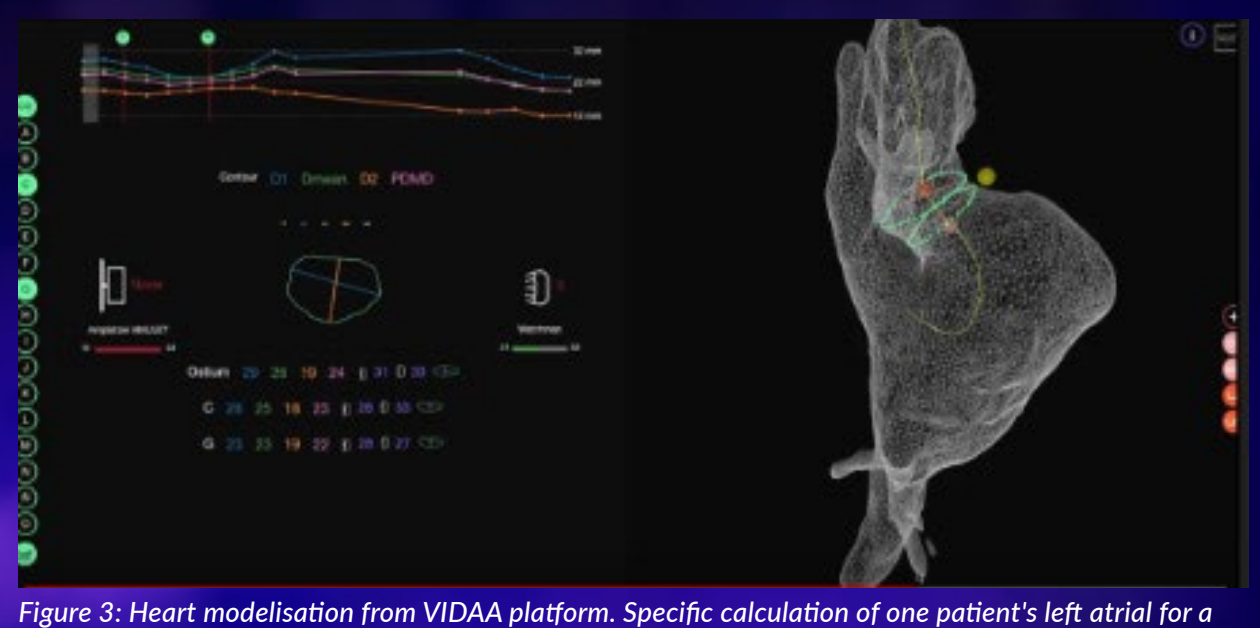

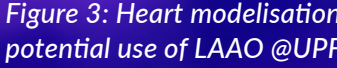

Scientists will run computer simulations likely to cause thrombus formation) to and validate their numerical models implant occluder devices. This will allow with observations from realistic physical us to model the entire treatment cycle phantoms of the heart and the vascular including thrombus formation, drug tree of interest created using 3D printing treatment models, and prediction of and soft robotics. They will also acquire treatment response.

experimental data of the heart's electrical activation with an optical fluorescence $\mathbf{3}$ Drugs safety, efficacy and technique.

2 Left atrial appendage occluder to reduce stroke risk.

The left atrial appendage (LAA) is a small vesicle located in the muscular wall of the left atrium. In atrial fibrillation, blood can form clots in this vicle, and when blod clots are pumped out of the heart, they can cause a stroke. To ovecome the risks, left a strick (LAAO) have been desisted to close the LAA in patents with atial fochlose to ormation of clots.

LAA occlusion is common in patients who are intolerant of anticoagulant therapy or who have bleeding risks associated with the use of such therapy.

Left atrial appendage occlusion reduces the risk of stroke in atrial fibrillation.

SimCardioTest uses 3D CT images and echocardiography to characterise the left atrial morphology (Figure 3) and simulate best configuration (i.e. one that is less

\section{cardiotoxicity}

The third study will assess drug safety and efficacy in personalised and detailed models. The model will allow for the measurement of the impact of different drug dosages mode will be buit at the celluar level and

These simulations will take into account both healthy and pathological conditions such as ischaemia (Figure 4), heart failure or atrial fibrillation, as well other patien characteristics like age and gender. A martphone could be used to easily track the outcome.

\section{In-silico trials}

On a cloud-based platform that is prestandardised, models for different pathologies can be tested. To quantify simulations will be performed that correspond to real measurements. The richness of the generated data could lead the development of new indices to efficacy/toxicity.
These simulations will be thoroughly certification process. This will be done in close collaboration with regulatory organisations.

In-silico trials can be used to develop time-to-market, and gain the trust

\section{Conclusion}

SimCardioTest will demonstrate the effectiveness of a cloud-based platform for new device and drug testing by combining computer modelling and simulation with $A$, using patient-specific data and HPC. This way, SimCardioTest aims to revolution cardiac research and facilitate the translation of therapies from the laboratory to the hospital and the patients.
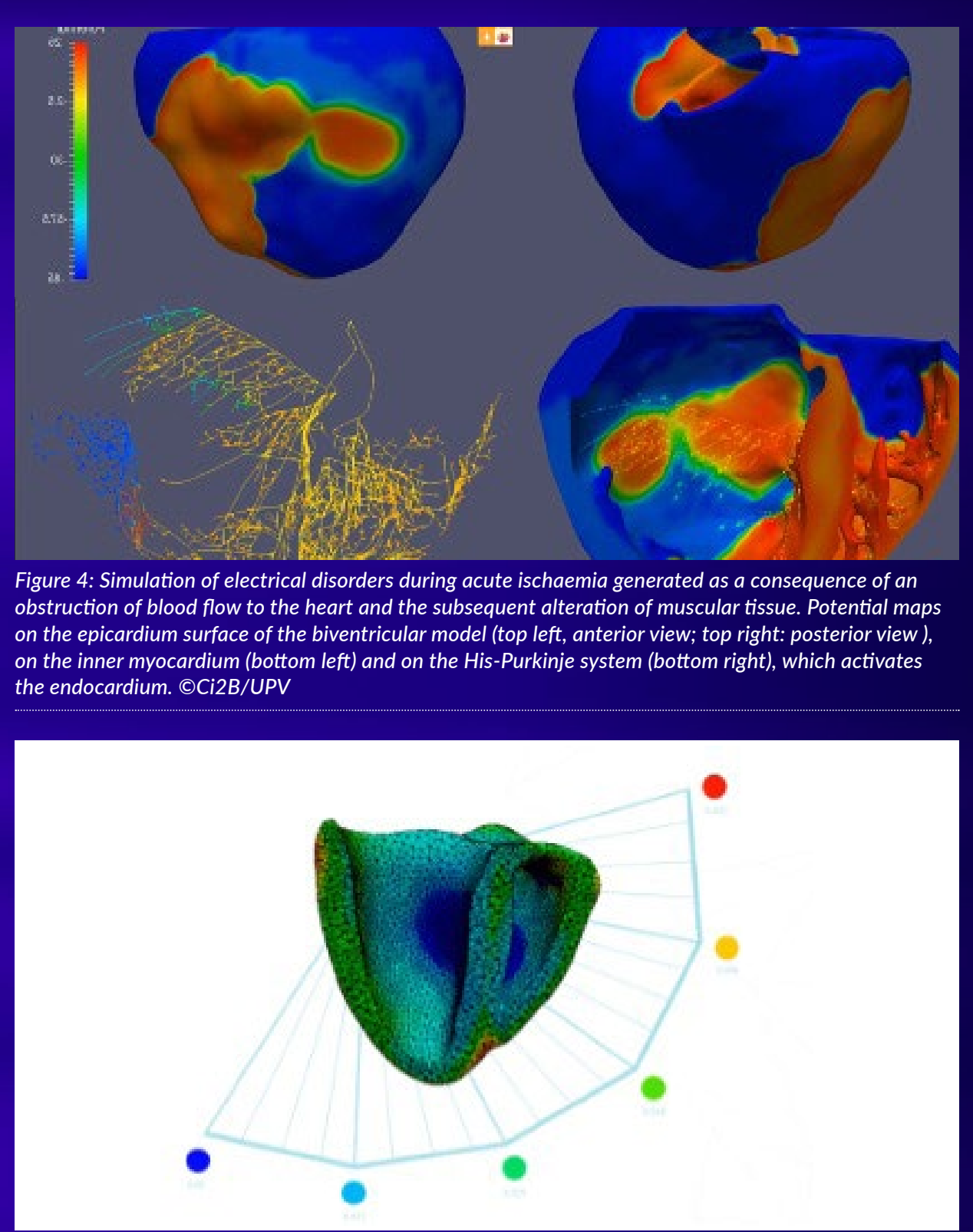

Figure 5: Simulation of beating heart. OGaetan Desrues/Inria. verified as the results can support the

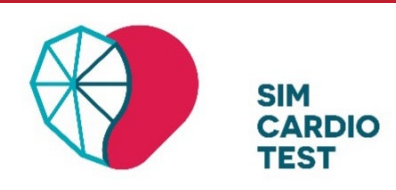

\section{PROJECT SUMMARY}

Cardiovascular diseases affect 15 million people in Europe, and digital solutions are now seen as very useful tools in the
search for new drugs and medical devices. search for new drugs and medical devices.
SimCardioTest is a four-year project funded by the European Commission that aims to develop credible computer modelling and platform for testing cardiac drugs and devices in silico.

\section{PROJECT LEAD}

SimCardioTest is led by Inria, the French national research institute for the digital sciences. A world-class research and technological support that create value in France and Europe. $\mathrm{Dr}$ Maxime Sermesant, Head of Computational Cardiology at Inria Epione and Chair of $\mathrm{Al}$ and Biophysics at 3IA Côte d'Azur, ensures the ased on pent characteristics. This gan level. This is a significant advance in the realism of the in-silico results,

\section{PROJECT PARTNERS}

SimCardioTest brings together leading experts in the field of cardiac simulation, (it) (Microport - CRM and Boston Scientific), University of Bordeaux, University Pompeu n international non-profit organisation (The Virtual Physiological Human Institute).

\section{CONTACT DETAILS}

Michèle Barbier

Inria Sophia Antipolis-Méditerranée,

2004 Route des Lucioles, 06902 Valbonne,

两 +33633079899

michele.barbier@inria.fr

https://www.simcardiotest.eu

in /SimCardioTest

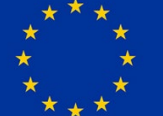

FUNDING

This project has received funding from the European
Union's Horizon 2020 research and innovation programme under grant agreement No.101016496. SMEs (ExactCure and InSilicoTrials), research organisations (Inria and Simula), universities

(1) @SimCardioTest 